\title{
APPROXIMATION BY LOCAL PARABOLIC SPLINES CONSTRUCTED ON THE BASIS OF INTERPOLATION IN THE MEAN ${ }^{1}$
}

\author{
Elena V. Strelkova \\ N.N. Krasovskii Institute of Mathematics and Mechanics, \\ Ural Branch of the Russian Academy of Sciences; \\ Ural Federal University, Ekaterinburg, Russia, \\ shevaldina@r66.ru
}

\begin{abstract}
The paper deals with approximative and form-retaining properties of the local parabolic splines of the form $S(x)=\sum_{j} y_{j} B_{2}(x-j h),(h>0)$, where $B_{2}$ is a normalized parabolic spline with the uniform nodes and functionals $y_{j}=y_{j}(f)$ are given for an arbitrary function $f$ defined on $\mathbb{R}$ by means of the equalities

$$
y_{j}=\frac{1}{h_{1}} \int_{\frac{-h_{1}}{2}}^{\frac{h_{1}}{2}} f(j h+t) d t \quad(j \in \mathbb{Z}) .
$$

On the class $W_{\infty}^{2}$ of functions under $0<h_{1} \leq 2 h$, the approximation error value is calculated exactly for the case of approximation by such splines in the uniform metrics.
\end{abstract}

Key words: Local parabolic splines, Approximation, Mean.

\section{Introduction}

In the function approximation theory, the local polynomial splines of the order $r$ and of minimal defect are usually constructed as linear combinations of the corresponding $B$-splines $B_{r, j}(x)$. For a function $f$ from the class of continuous ones, the local polynomial spline $S(x)=S(f, x)$ is defined as follows:

$$
S(x)=\sum_{j} b_{j}(f) B_{r, j}(x)
$$

where $b_{j}(f)$ is the sequence of linear continuous functionals, whose choice determines the form of the approximation.

As the functionals $b_{j}(f)$, one chooses the linear combinations of the function values and its derivatives at the mesh nodes or its divided differences.

The most simple and convenient (in computation) version of this choice is $b_{j}(f)=f\left(x_{j}\right)$ (here, $x_{j}$ are the nodes of the spline $S$ mesh). It leads to the well known local spline (see, for example, $[1-4])$ :

$$
S(x)=\sum_{j} f\left(x_{j}\right) B_{r, j}(x) .
$$

In formula (0.2) instead of $x_{j}$, the arithmetic mean is often used that is calculated over all nodes of the $B$-spline $B_{r, j}(x)([1-3])$.

\footnotetext{
${ }^{1}$ The paper was originally published in Trudy Institute of Mathematics and Mechanics, Ural Branch of the Russian Academy of Sciences, 2007. Vol. 13, no. 4. P. 169-189 (in Russian).
} 
A spline (constructed in such a way) is not an interpolation one. But in the case $r=2$ (i.e., of the parabolic splines), it is a continuously differentiable function on the whole number axis $\mathbb{R}$ and possesses both the form-retaining and extremal features [5].

But if the function $f$ is not continuous but only integrable, it is not natural to consider aspects of this function approximation taking into account only its values at the mesh nodes. It is so since its values at separate points are not essential for functions of such a type. In such case, one uses the interpolation on the average. Aspects of existence, uniqueness, and approximative and extremal properties of such splines were investigated in works [6-8]. (Generalizations onto the $L$-splines see, also, on $[9,10])$.

Let two real numbers $h>0$ and $h_{1}>0$ be given. For a function integrable on the whole number axis $f: \mathbb{R} \rightarrow \mathbb{R}$ assume

$$
b_{j}(f)=y_{j}=\left\{\begin{array}{cl}
\frac{1}{h_{1}} \int_{-\frac{h_{1}}{2}}^{\frac{h_{1}}{2}} f(j h+t) d t, & h_{1}>0, \\
f(j h), & h_{1}=0 .
\end{array}\right.
$$

Let $B_{2,0}(x)$ be a normalized parabolic $B$-spline (see, for example, [1]) with nodes $-\frac{3 h}{2},-\frac{h}{2}, \frac{h}{2}, \frac{3 h}{2}$, and $B_{2, j}(x)=B_{2,0}(x-j h)$.

Let, also, $W_{\infty}^{2}=W_{\infty}^{2}(\mathbb{X})=\left\{f: f^{\prime} \in A C,\left\|f^{\prime \prime}\right\|_{\infty} \leq 1\right\}$ be a class of functions given on the set $\mathbb{X}(\mathbb{X}=\mathbb{R}$ or $\mathbb{X}=[a, b])$.

Here, $A C$ is the class of locally absolute continuous functions $\|\cdot\|_{\infty}=\|\cdot\|_{L_{\infty}(\mathbb{X})}, L_{\infty}(\mathbb{X})$ is the class of the functions essentially restricted on $\mathbb{X}$ with the usual definition of the norm

$$
\|f\|_{\infty}=\operatorname{esssup}_{x \in \mathbb{X}}|f(x)|
$$

In the present work, we investigate in details aspects of approximation of smooth functions $f$ by the local parabolic splines of the form (0.1) (i.e., for $r=2$ ) with the choice of the functionals $b_{j}(f)$ in the form $(0.3)$.

\section{Properties of the spline $S$}

On the axis $\mathbb{R}$ (on both its sides), consider the following mesh of nodes: $\cdots<x_{-2}<x_{-1}<$ $x_{0}<x_{1}<x_{2}<\cdots ;$ and let $x_{j}=j h, h=x_{j+1}-x_{j}, \quad x_{j+1 / 2}=x_{j}+\frac{h}{2}(j \in \mathbb{Z})$.

For $x_{j} \leq x \leq x_{j+1 / 2}$ from formulas (0.1) and (0.3), it follows

$$
\begin{aligned}
S(x)=y_{j-1} \cdot & \frac{\left(x-x_{j+1 / 2}\right)^{2}}{2 h^{2}}+y_{j} \cdot\left(\frac{x_{j+1}-x}{h}-\frac{\left(x-x_{j+1 / 2}\right)^{2}}{h^{2}}\right) \\
& +y_{j+1} \cdot\left(\frac{\left(x-x_{j+1 / 2}\right)^{2}}{2 h^{2}}+\frac{x-x_{j}}{h}\right),
\end{aligned}
$$

and for $x_{j+1 / 2} \leq x \leq x_{j+1}$

$$
\begin{gathered}
S(x)=y_{j} \cdot\left(\frac{\left(x-x_{j+1 / 2}\right)^{2}}{2 h^{2}}+\frac{x_{j+1}-x}{h}\right)+y_{j+1} \cdot\left(\frac{x-x_{j}}{h}-\frac{\left(x-x_{j+1 / 2}\right)^{2}}{h^{2}}\right) \\
+y_{j+2} \cdot \frac{\left(x-x_{j+1 / 2}\right)^{2}}{2 h^{2}} .
\end{gathered}
$$


To the function $f(x) \in W_{\infty}^{2}(\mathbb{R})$, we put in correspondence the parabolic spline $S(x)=S(f, x)$ (see (1.1)-(1.2)), where the functionals $y_{j}$ are defined by formula (0.3).

For $h_{1}=0$, the form-retaining and approximation properties of such splines were investigated in [5]; so, we shall consider the case $h_{1}>0$.

Denote by $A_{j}$ the interval $\left(x_{j}-\frac{h_{1}}{2} ; x_{j}+\frac{h_{1}}{2}\right), j \in \mathbb{Z}$.

Theorem 1. The local spline $S(x)$ defined by formulas (1.1)-(1.2), possesses the following properties:

1) locally inherits the sign of the original function $f$ in the sense that

a) if $f(x) \geq 0(\leq 0)$ for $x \in A_{j-1} \bigcup A_{j} \bigcup A_{j+1}$, then $S(x) \geq 0(\leq 0)$ for $x_{j} \leq x \leq x_{j+1 / 2}(j \in \mathbb{Z})$;

b) if $f(x) \geq 0(\leq 0)$ for $x \in A_{j} \bigcup A_{j+1} \bigcup A_{j+2}$, then $S(x) \geq 0(\leq 0)$ for $x_{j+1 / 2} \leq x \leq x_{j+1}(j \in \mathbb{Z})$;

2) locally inherits the monotonicity property of the original function $f$, namely,

a) if the function $f(x)$ does not decrease (does not increase) in the interval $\left(x_{j-1}-\frac{h_{1}}{2} ; x_{j+1}+\frac{h_{1}}{2}\right)$, then the spline $S(x)$ does not decrease (does not increase) in the interval $\left(x_{j} ; x_{j+1 / 2}\right)(j \in \mathbb{Z})$;

$b)$ if the function $f(x)$ does not decrease (does not increase) in the interval $\left(x_{j}-\frac{h_{1}}{2} ; x_{j+2}+\frac{h_{1}}{2}\right)$, then the spline $S(x)$ does not decrease (does not increase) in the interval $\left(x_{j+1 / 2} ; x_{j+1}\right)(j \in \mathbb{Z})$.

P r o o f. For the point 1 ), the proof follows directly from non-negativity of the $B$-spline $B_{2,0}(x)$, formula (0.2), and non-negativity of $y_{j}$ for $x \in A_{j}$.

Point 2a). From condition of point 2) of Theorem 1 and definition of $y_{j}$ it follows $y_{j+1} \geq y_{j} \geq$ $y_{j-1}$. By differentiation of the right-hand side of equations (1.1), we obtain that for $x \in\left(x_{j} ; x_{j+1 / 2}\right)$ the derivative

$$
S^{\prime}(x)=\frac{y_{j+1}-y_{j}}{h}+\frac{x-x_{j+1 / 2}}{h^{2}} \cdot\left(y_{j+1}-2 y_{j}+y_{j+1}\right)
$$

of the spline $S$ in this interval is a linear function in the variable $x$. So, to prove point 2 ), it is sufficient to verify that for $y_{j+1} \geq y_{j} \geq y_{j-1}$, inequalities $S^{\prime}\left(x_{j}+0\right) \geq 0, S^{\prime}\left(x_{j+1 / 2}-0\right) \geq 0$ hold. Validity of these inequalities follows from the formulas

$$
\begin{gathered}
S^{\prime}\left(x_{j}+0\right)=\frac{y_{j+1}-y_{j-1}}{2 h}, \\
S^{\prime}\left(x_{j+1 / 2}-0\right)=\frac{y_{j+1}-y_{j}}{h}
\end{gathered}
$$

and point $2 \mathrm{a}$ ) of Theorem 1 is proved.

Point $\mathbf{2 b}$ ). From conditions of point $2 \mathrm{~b}$ ) of Theorem 1 and definition of $y_{j}$, it follows $y_{j+2} \geq$ $y_{j+1} \geq y_{j}$. By differentiation of the right-hand side of equality (1.2), we obtain that for $x \in$ $\left(x_{j+1 / 2} ; x_{j}\right)$, the derivative

$$
S^{\prime}(x)=\frac{y_{j+1}-y_{j}}{h}+\frac{x-x_{j+1 / 2}}{h^{2}} \cdot\left(y_{j+2}-2 y_{j+1}+y_{j}\right)
$$

of the spline $S$ in this interval is a linear function in the variable $x$. So, to prove point $\mathrm{b}$ ), it is sufficient to verify that for $y_{j+2} \geq y_{j+1} \geq y_{j}$, the equalities $S^{\prime}\left(x_{j+1 / 2}+0\right) \geq 0, S^{\prime}\left(x_{j+1}-0\right) \geq 0$ hold. But this follows from the formulae

$$
\begin{aligned}
S^{\prime}\left(x_{j+1 / 2}+0\right) & =\frac{y_{j+1}-y_{j}}{h}, \\
S^{\prime}\left(x_{j+1}-0\right) & =\frac{y_{j+2}-y_{j}}{2 h} .
\end{aligned}
$$


Before formulation of further statements, obtain the integral representation for the difference $S(x)-f(x)$ in the interval $\left[x_{j} ; x_{j+1}\right]$ for $0<h_{1} \leq 2 h$.

Let for the beginning $x \in\left[x_{j} ; x_{j+1 / 2}\right]$. Then, by the Taylor formula for the function $f(x) \in$ $W_{\infty}^{2}(\mathbb{R})$ we have

$$
f(x)=f\left(x_{j}\right)+f^{\prime}\left(x_{j}\right)\left(x-x_{j}\right)+\int_{x_{j}}^{x}(x-t) f^{\prime \prime}(t) d t .
$$

Using (1.3) and definition $y_{j}$ (see (0.3)), we derive

$$
\begin{gathered}
y_{j-1}=f\left(x_{j}\right)-f^{\prime}\left(x_{j}\right) h+\frac{1}{h_{1}} \int_{-\frac{h_{1}}{2}}^{\frac{h_{1}}{2}} d s \int_{x_{j}}^{s+x_{j-1}}\left(x_{j-1}+s-t\right) f^{\prime \prime}(t) d t, \\
y_{j}=f\left(x_{j}\right)+\frac{1}{h_{1}} \int_{-\frac{h_{1}}{2}}^{\frac{h_{1}}{2}} d s \int_{x_{j}}^{s+x_{j}}\left(x_{j}+s-t\right) f^{\prime \prime}(t) d t, \\
y_{j+1}=f\left(x_{j}\right)+f^{\prime}\left(x_{j}\right) h+\frac{1}{h_{1}} \int_{-\frac{h_{1}}{2}}^{\frac{h_{1}}{2}} d s \int_{x_{j}}^{s+x_{j+1}}\left(x_{j+1}+s-t\right) f^{\prime \prime}(t) d t .
\end{gathered}
$$

Therefore, from (1.1), we obtain

$$
\begin{gathered}
S(x)=\frac{\left(x-x_{j+1 / 2}\right)^{2}}{2 h^{2}}\left[f\left(x_{j}\right)-f^{\prime}\left(x_{j}\right) h+\frac{1}{h_{1}} \int_{-\frac{h_{1}}{2}}^{\frac{h_{1}}{2}} d s \int_{x_{j}}^{s+x_{j-1}}\left(x_{j-1}+s-t\right) f^{\prime \prime}(t) d t\right] \\
+\left(\frac{x_{j+1}-x}{h}-\frac{\left(x-x_{j+1 / 2}\right)^{2}}{h^{2}}\right)\left[f\left(x_{j}\right)+\frac{1}{h_{1}} \int_{-\frac{h_{1}}{2}}^{\frac{h_{1}}{2}} d s \int_{x_{j}}^{s+x_{j}}\left(x_{j}+s-t\right) f^{\prime \prime}(t) d t\right] \\
+\left(\frac{\left(x-x_{j+1 / 2}\right)^{2}}{2 h^{2}}+\frac{x-x_{j}}{h}\right)\left[f\left(x_{j}\right)+f^{\prime}\left(x_{j}\right) h+\frac{1}{h_{1}} \int_{-\frac{h_{1}}{2}}^{\frac{h_{1}}{2}} d s \int_{x_{j}}^{s+x_{j+1}}\left(x_{j+1}+s-t\right) f^{\prime \prime}(t) d t\right] .
\end{gathered}
$$

Taking into account that $0<h_{1} \leq 2 h$, change the integration order in the integrals entering 
into representation (1.4). Hence, we obtain

$$
\begin{gathered}
S(x)=\frac{\left(x-x_{j+1 / 2}\right)^{2}}{2 h^{2}}\left\{f\left(x_{j}\right)-f^{\prime}\left(x_{j}\right) h+\frac{1}{h_{1}}\left[\int_{x_{j-1}-\frac{h_{1}}{2}}^{x_{j-1}+\frac{h_{1}}{2}} \frac{1}{2} f^{\prime \prime}(t)\left(t-x_{j-1}+\frac{h_{1}}{2}\right)^{2} d t\right.\right. \\
\left.\left.+\int_{x_{j-1}+\frac{h_{1}}{2}}^{x_{j}} f^{\prime \prime}(t) h_{1}\left(t-x_{j-1}\right) d t\right]\right\}+\left(\frac{x_{j+1}-x}{h}-\frac{\left(x-x_{j+1 / 2}\right)^{2}}{h^{2}}\right) \\
\times\left\{f\left(x_{j}\right)+\frac{1}{h_{1}}\left[\int_{x_{j}-\frac{h_{1}}{2}}^{x_{j}} \frac{1}{2} f^{\prime \prime}(t)\left(t-x_{j}+\frac{h_{1}}{2}\right)^{2} d t+\int_{x_{j}}^{x_{j}+\frac{h_{1}}{2}} \frac{1}{2} f^{\prime \prime}(t)\left(t-x_{j}-\frac{h_{1}}{2}\right)^{2} d t\right]\right\} \\
+\left(\frac{\left(x-x_{j+1 / 2}\right)^{2}}{2 h^{2}}+\frac{x-x_{j}}{h}\right)\left\{f\left(x_{j}\right)+f^{\prime}\left(x_{j}\right) h+\frac{1}{h_{1}}\left[\int_{x_{j}}^{x_{j+1}-\frac{h_{1}}{2}} f^{\prime \prime}(t) h_{1}\left(x_{j+1}-t\right) d t\right.\right. \\
\left.\left.+\int_{x_{j+1}+\frac{h_{1}}{2}}^{x_{j+1}-\frac{h_{1}}{2}} \frac{1}{2} f^{\prime \prime}(t)\left(t-x_{j+1}-\frac{h_{1}}{2}\right)^{2} d t\right]\right\}, \quad x \in\left[x_{j} ; x_{j+1 / 2}\right] .
\end{gathered}
$$

By virtue of symmetry of formulas for $S(x)$ w.r.t. the middle $x_{j+1 / 2}$ of the interval $\left[x_{j} ; x_{j+1}\right]$, we obtain the following similar representation of $S(x)$ for $x \in\left[x_{j+1 / 2}, x_{j+1}\right]$ :

$$
\begin{gathered}
S(x)=\left(\frac{\left(x-x_{j+1 / 2}\right)^{2}}{2 h^{2}}+\frac{x_{j+1}-x}{h}\right)\left\{f\left(x_{j}\right)+\frac{1}{h_{1}}\left[\int_{x_{j}-\frac{h_{1}}{2}}^{x_{j}} \frac{1}{2} f^{\prime \prime}(t)\left(t-x_{j}+\frac{h_{1}}{2}\right)^{2} d t\right.\right. \\
\left.\left.+\int_{x_{j}}^{x_{j}+\frac{h_{1}}{2}} \frac{1}{2} f^{\prime \prime}(t)\left(t-x_{j}-\frac{h_{1}}{2}\right)^{2} d t\right]\right\}+\left(\frac{x-x_{j}}{h}-\frac{\left(x-x_{j+1 / 2}\right)^{2}}{h^{2}}\right)\left\{f\left(x_{j}\right)+f^{\prime}\left(x_{j}\right) h\right. \\
\left.+\frac{1}{h_{1}}\left[\int_{x_{j}}^{x_{j+1}-\frac{h_{1}}{2}} f^{\prime \prime}(t) h_{1}\left(x_{j+1}-t\right) d t+\int_{x_{j+1}-\frac{h_{1}}{2}}^{x_{j+1}+\frac{h_{1}}{2}} \frac{1}{2} f^{\prime \prime}(t)\left(t-x_{j+1}-\frac{h_{1}}{2}\right)^{2} d t\right]\right\} \\
+\frac{\left(x-x_{j+1 / 2}\right)^{2}}{2 h^{2}}\left\{f\left(x_{j}\right)+f^{\prime}\left(x_{j}\right) 2 h+\frac{1}{h_{1}}\left[\int_{x_{j}}^{x_{j+2}-\frac{h_{1}}{2}} f^{\prime \prime}(t) h_{1}\left(x_{j+2}-t\right) d t\right.\right. \\
\left.\left.+\int_{x_{j+2}+\frac{h_{1}}{2}}^{1} \frac{1}{2} f^{\prime \prime}(t)\left(t-x_{j+2}-\frac{h_{1}}{2}\right)^{2} d t\right]\right\}, \quad x \in\left[x_{j+1 / 2}, x_{j+1}\right] .
\end{gathered}
$$

Theorem 2. A local spline $S(x)$ defined by formula (1.1) - (1.2), for $0<h_{1} \leq 2 h$, possesses the following properties: 
1) inherits locally the convexity property of the original function $f$, namely,

a) if the function $f(x)$ is down- (upper) convex in the interval $\left(x_{j-1}-\frac{h_{1}}{2} ; x_{j+1}+\frac{h_{1}}{2}\right)$, then the spline $S(x)$ is the down- (upper-) convex function in the interval $\left(x_{j} ; x_{j+1 / 2}\right)(j \in \mathbb{Z})$;

$b)$ if the function $f(x)$ is down- (upper-) convex in the interval $\left(x_{j}-\frac{h_{1}}{2} ; x_{j+2}+\frac{h_{1}}{2}\right)$, then the spline $S(x)$ is the down- (upper-) convex function in the interval $\left(x_{j+1 / 2} ; x_{j+1}\right)(j \in \mathbb{Z})$.

2) a) for any function $f \in W_{\infty}^{2}\left[x_{j-1}-\frac{h_{1}}{2} ; x_{j+1}+\frac{h_{1}}{2}\right]$, the exact inequality holds

$$
\left|S^{\prime \prime}(x)\right| \leq 1, \quad x \in\left(x_{j} ; x_{j+1 / 2}\right) \text {, }
$$

and, moreover, for all $x \in\left(x_{j} ; x_{j+1 / 2}\right)$, the inequality sign is provided by the function $f(x)=\frac{x^{2}}{2}$;

b) for any function $f \in W_{\infty}^{2}\left[x_{j}-\frac{h_{1}}{2} ; x_{j+2}+\frac{h_{1}}{2}\right]$, the exact inequality holds

$$
\left|S^{\prime \prime}(x)\right| \leq 1, \quad x \in\left(x_{j+1 / 2} ; x_{j+1}\right) \text {, }
$$

and, moreover, for all $x \in\left(x_{j+1 / 2} ; x_{j+1}\right)$, the inequality sign is provided by the function $f(x)=\frac{x^{2}}{2}$.

P r o o f. To prove $1 a$ ), it is necessary to verify that if

$$
f^{\prime \prime}(x) \geq 0(\leq 0) \quad \text { for } \quad x \in\left(x_{j-1}-\frac{h_{1}}{2} ; x_{j+1}+\frac{h_{1}}{2}\right),
$$

then $S^{\prime \prime}(x) \geq 0(\leq 0)$ for $x \in\left(x_{j} ; x_{j+1 / 2}\right)$.

By the twice differentiation of the function $S(x)$, we obtain from formula (1.5)

$$
\begin{gathered}
S^{\prime \prime}(x)=\int_{x_{j-1}-\frac{h_{1}}{2}}^{x_{j-1}+\frac{h_{1}}{2}} f^{\prime \prime}(t) C_{1}(t) d t+\int_{x_{j-1}+\frac{h_{1}}{2}}^{x_{j}} f^{\prime \prime}(t) C_{2}(t) d t+\int_{x_{j}-\frac{h_{1}}{2}}^{x_{j}} f^{\prime \prime}(t) C_{3}(t) d t \\
+\int_{x_{j}}^{x_{j}+\frac{h_{1}}{2}} f^{\prime \prime}(t) C_{4}(t) d t+\int_{x_{j}}^{x_{j+1}-\frac{h_{1}}{2}} f^{\prime \prime}(t) C_{5}(t) d t+\int_{x_{j+1}-\frac{h_{1}}{2}}^{x_{j+1}+\frac{h_{1}}{2}} f^{\prime \prime}(t) C_{6}(t) d t,
\end{gathered}
$$

where

$$
\begin{aligned}
& C_{1}(t)=\frac{1}{2 h^{2} h_{1}}\left(t-x_{j-1}+\frac{h_{1}}{2}\right)^{2}, \quad C_{2}(t)=\frac{1}{h^{2}}\left(t-x_{j-1}\right), \quad C_{3}(t)=-\frac{1}{h^{2} h_{1}}\left(t-x_{j}+\frac{h_{1}}{2}\right)^{2}, \\
& C_{4}(t)=-\frac{1}{h^{2} h_{1}}\left(t-x_{j}-\frac{h_{1}}{2}\right)^{2}, \quad C_{5}(t)=\frac{1}{h^{2}}\left(x_{j+1}-t\right), \quad C_{6}(t)=\frac{1}{2 h^{2} h_{1}}\left(t-x_{j+1}-\frac{h_{1}}{2}\right)^{2} .
\end{aligned}
$$

Divide the further proof into two cases: 1) $0<h_{1} \leq h$, and 2) $h<h_{1} \leq 2 h$.

Case 1). Let $0<h_{1} \leq h$. Under this, the function $S^{\prime \prime}(x)$ is transformed to the form

$$
\begin{gathered}
S^{\prime \prime}(x)=\int_{x_{j-1}-\frac{h_{1}}{2}}^{x_{j-1}+\frac{h_{1}}{2}} f^{\prime \prime}(t) C_{1}(t) d t+\int_{x_{j-1}+\frac{h_{1}}{2}}^{x_{j}-\frac{h_{1}}{2}} f^{\prime \prime}(t) C_{2}(t) d t+\int_{x_{j}-\frac{h_{1}}{2}}^{x_{j}} f^{\prime \prime}(t)\left(C_{2}(t)+C_{3}(t)\right) d t \\
+\int_{x_{j}}^{x_{j}+\frac{h_{1}}{2}} f^{\prime \prime}(t)\left(C_{4}(t)+C_{5}(t)\right) d t+\int_{x_{j}+\frac{h_{1}}{2}}^{x_{j+1}-\frac{h_{1}}{2}} f^{\prime \prime}(t) C_{5}(t) d t+\int_{x_{j+1}-\frac{h_{1}}{2}}^{x_{1}} f^{\prime \prime}(t) C_{6}(t) d t .
\end{gathered}
$$


From the definitions of $C_{j}(t)(j=\overline{1,6})$, it follows that $C_{1}(t) \geq 0$ for $t \in\left[x_{j-1}-\frac{h_{1}}{2} ; x_{j-1}+\frac{h_{1}}{2}\right]$, $C_{2}(t) \geq 0$ for $t \in\left[x_{j-1}+\frac{h_{1}}{2} ; x_{j}-\frac{h_{1}}{2}\right], C_{5}(t) \geq 0$ for $t \in\left[x_{j}+\frac{h_{1}}{2} ; x_{j+1}-\frac{h_{1}}{2}\right]$, and $C_{6}(t) \geq 0$ for $t \in\left[x_{j+1}-\frac{h_{1}}{2} ; x_{j+1}+\frac{h_{1}}{2}\right]$.

Now it remains to investigate the quadratic trinomials $C_{2}(t)+C_{3}(t)$ for $t \in\left[x_{j}-\frac{h_{1}}{2} ; x_{j}\right]$ and $C_{4}(t)+C_{5}(t)$ for $t \in\left[x_{j} ; x_{j}+\frac{h_{1}}{2}\right]$.

Non-negativity of the functions $C_{2}(t)+C_{3}(t)$ for $t \in\left[x_{j}-\frac{h_{1}}{2} ; x_{j}\right]$ and $C_{4}(t)+C_{5}(t)$ for $t \in\left[x_{j} ; x_{j}+\frac{h_{1}}{2}\right]$ follows from the fact that the branches of corresponding parabolas are downdirected and their values (at the ends of the intervals under investigation) are non-negative. Namely,

$$
\begin{gathered}
C_{2}\left(x_{j}\right)+C_{3}\left(x_{j}\right)=C_{4}\left(x_{j}\right)+C_{5}\left(x_{j}\right)=\frac{4 h-h_{1}}{4 h^{2}} \geq 0, \\
C_{2}\left(x_{j}-\frac{h_{1}}{2}\right)+C_{3}\left(x_{j}-\frac{h_{1}}{2}\right)=C_{4}\left(x_{j}+\frac{h_{1}}{2}\right)+C_{5}\left(x_{j}+\frac{h_{1}}{2}\right)=\frac{2 h-h_{1}}{2 h^{2}} \geq 0 .
\end{gathered}
$$

From the statements proved, representation (1.7), and the condition $f^{\prime \prime}(t) \geq 0$ for $t \in\left(x_{j-1}-\frac{h_{1}}{2} ; x_{j+1}+\frac{h_{1}}{2}\right)$, it follows that $S^{\prime \prime}(x) \geq 0$ for $x \in\left(x_{j} ; x_{j+1 / 2}\right)$.

Case 2). Let $h<h_{1} \leq 2 h$. Under this, the function $S^{\prime \prime}(x)$ is transformed to the form

$$
\begin{gathered}
S^{\prime \prime}(x)=\int_{x_{j-1}-\frac{h_{1}}{2}}^{x_{j}-\frac{h_{1}}{2}} f^{\prime \prime}(t) C_{1}(t) d t+\int_{x_{j}-\frac{h_{1}}{2}}^{x_{j-1}+\frac{h_{1}}{2}} f^{\prime \prime}(t)\left(C_{1}(t)+C_{3}(t)\right) d t+\int_{x_{j-1}+\frac{h_{1}}{2}}^{x_{j}} f^{\prime \prime}(t)\left(C_{2}(t)+C_{3}(t)\right) d t \\
+\int_{x_{j}}^{x_{j+1}-\frac{h_{1}}{2}} f^{\prime \prime}(t)\left(C_{4}(t)+C_{5}(t)\right) d t+\int_{x_{j+1}-\frac{h_{1}}{2}}^{x_{j}+\frac{h_{1}}{2}} f^{\prime \prime}(t)\left(C_{4}(t)+C_{6}(t)\right) d t+\int_{x_{j}+\frac{h_{1}}{2}}^{x_{2}} f^{\prime \prime}(t) C_{6}(t) d t .
\end{gathered}
$$

From the definitions of $C_{j}(t)(j=\overline{1,6})$, it follows that $C_{1}(t) \geq 0$ for $t \in\left[x_{j-1}-\frac{h_{1}}{2} ; x_{j}-\frac{h_{1}}{2}\right]$ and $C_{6}(t) \geq 0$ for $t \in\left[x_{j}+\frac{h_{1}}{2} ; x_{j+1}+\frac{h_{1}}{2}\right]$.

Elementary calculations show that

$$
\begin{gathered}
C_{1}\left(x_{j}-\frac{h_{1}}{2}\right)+C_{3}\left(x_{j}-\frac{h_{1}}{2}\right)=C_{4}\left(x_{j}+\frac{h_{1}}{2}\right)+C_{6}\left(x_{j}+\frac{h_{1}}{2}\right)=\frac{1}{2 h_{1}} \geq 0, \\
C_{1}\left(x_{j-1}+\frac{h_{1}}{2}\right)+C_{3}\left(x_{j-1}+\frac{h_{1}}{2}\right)=C_{4}\left(x_{j+1}+\frac{h_{1}}{2}\right)+C_{6}\left(x_{j+1}+\frac{h_{1}}{2}\right)=\frac{2 h^{2}-\left(h_{1}-2 h\right)^{2}}{2 h_{1} h^{2}} \geq 0, \\
C_{2}\left(x_{j}\right)+C_{3}\left(x_{j}\right)=C_{4}\left(x_{j}\right)+C_{5}\left(x_{j}\right)=\frac{1}{h^{2}}\left(h-\frac{h_{1}}{4}\right) \geq 0, \\
C_{2}\left(x_{j-1}+\frac{h_{1}}{2}\right)+C_{3}\left(x_{j-1}+\frac{h_{1}}{2}\right)=C_{4}\left(x_{j+1}-\frac{h_{1}}{2}\right)+C_{5}\left(x_{j+1}-\frac{h_{1}}{2}\right)=\frac{2 h^{2}-\left(h_{1}-2 h\right)^{2}}{2 h_{1} h^{2}} \geq 0 .
\end{gathered}
$$

Since the quadratic trinomials $C_{1}(t)+C_{3}(t), C_{2}(t)+C_{3}(t), C_{4}(t)+C_{5}(t)$, and $C_{4}(t)+C_{6}(t)$ have negative leading coefficients and their values at the ends of the corresponding intervals are 
non-negative, we have

$$
\begin{gathered}
C_{1}(t)+C_{3}(t) \geq 0 \text { for } t \in\left[x_{j}-\frac{h_{1}}{2} ; x_{j-1}+\frac{h_{1}}{2}\right], \\
C_{2}(t)+C_{3}(t) \geq 0 \text { for } t \in\left[x_{j-1}+\frac{h_{1}}{2} ; x_{j}\right], \\
C_{4}(t)+C_{5}(t) \geq 0 \text { for } t \in\left[x_{j} ; x_{j+1}-\frac{h_{1}}{2}\right], \\
C_{4}(t)+C_{6}(t) \geq 0 \text { for } t \in\left[x_{j+1}-\frac{h_{1}}{2} ; x_{j}+\frac{h_{1}}{2}\right] .
\end{gathered}
$$

From the statements proved, representation (1.8), and the condition $f^{\prime \prime}(t) \geq 0$ for $t \in\left(x_{j-1}-\right.$ $\left.\frac{h_{1}}{2} ; x_{j+1}+\frac{h_{1}}{2}\right)$, it follows that $S^{\prime \prime}(x) \geq 0$ for $x \in\left(x_{j} ; x_{j+1 / 2}\right)$.

Proof of point $1 \mathbf{b}$ ) one-to-one repeats the reasonings mentioned in the prof of point $1 a$ ) after substitution the variable $x-x_{j}$ by the $x_{j+1}-x$ one.

Point 2a). Estimate $\left|S^{\prime \prime}(x)\right|$ for $x \in\left[x_{j} ; x_{j+1 / 2}\right]$. Under $0<h_{1} \leq h$ by (1.7) for any function $f \in W_{\infty}^{2}\left[x_{j-1}-\frac{h_{1}}{2} ; x_{j+1}+\frac{h_{1}}{2}\right]$, the value $\left|S^{\prime \prime}(x)\right|$ is estimated from above by the sum of integrals, and this sum is equal to 1 . Namely,

$$
\begin{gathered}
\left|S^{\prime \prime}(x)\right| \leq \int_{x_{j-1}-\frac{h_{1}}{2}}^{x_{j-1}+\frac{h_{1}}{2}} C_{1}(t) d t+\int_{x_{j-1}+\frac{h_{1}}{2}}^{x_{j}-\frac{h_{1}}{2}} C_{2}(t) d t+\int_{x_{j}-\frac{h_{1}}{2}}^{x_{j}}\left(C_{2}(t)+C_{3}(t)\right) d t \\
+\int_{x_{j}}^{x_{j}+\frac{h_{1}}{2}}\left(C_{4}(t)+C_{5}(t)\right) d t+\int_{x_{j}+\frac{h_{1}}{2}}^{x_{j+1}-\frac{h_{1}}{2}} C_{5}(t) d t+\int_{x_{j+1}-\frac{h_{1}}{2}}^{x_{j+1}+\frac{h_{1}}{2}} C_{6}(t) d t=1, \quad x \in\left[x_{j} ; x_{j+1 / 2}\right] .
\end{gathered}
$$

Similarly, for $h<h_{1} \leq 2 h$ from (1.7), we derive that for any function $f \in W_{\infty}^{2}\left[x_{j}-\frac{h_{1}}{2} ; x_{j+2}+\frac{h_{1}}{2}\right]$ for $x \in\left[x_{j} ; x_{j+1 / 2}\right]$, the following inequality holds:

$$
\begin{gathered}
\left|S^{\prime \prime}(x)\right| \leq \int_{x_{j-1}-\frac{h_{1}}{2}}^{x_{j}-\frac{h_{1}}{2}} C_{1}(t) d t+\int_{x_{j}-\frac{h_{1}}{2}}^{x_{j-1}+\frac{h_{1}}{2}}\left(C_{1}(t)+C_{3}(t)\right) d t+\int_{x_{j-1}+\frac{h_{1}}{2}}^{x_{j}}\left(C_{2}(t)+C_{3}(t)\right) d t \\
+\int_{x_{j}}^{x_{j+1}-\frac{h_{1}}{2}}\left(C_{4}(t)+C_{5}(t)\right) d t+\int_{x_{j+1}-\frac{h_{1}}{2}}^{x_{j+1}+\frac{h_{1}}{2}}\left(C_{4}(t)+C_{6}(t)\right) d t+\int_{x_{j}+\frac{h_{1}}{2}}^{x_{2}} C_{6}(t) d t=1 .
\end{gathered}
$$

From the proofs considered above, it follows that in both cases the function that realizes exact equality in the inequalities proved is the function $f(x)=\frac{x^{2}}{2}$.

Point $\mathbf{2 b}$ ). The proof follows directly from the function $S(x)$ symmetry w.r.t. the middle point $x_{j+1 / 2}$ of the interval $\left[x_{j} ; x_{j+1}\right]$. 


\section{Estimations of approximation errors}

Theorem 3. For $0 \leq h_{1} \leq 2 h$, the following equality holds:

$$
\sup _{f \in W_{\infty}^{2}(\mathbb{R})}\|f-S\|_{\infty}=\frac{h^{2}}{8}+\frac{h_{1}^{2}}{24} .
$$

P r o o f. Consider for $x \in\left[x_{j} ; x_{j+1 / 2}\right]$ non-integral terms in the function $f \in W_{\infty}^{2}\left[x_{j-1}-\right.$ $\left.\frac{h_{1}}{2} ; x_{j+1}+\frac{h_{1}}{2}\right]$ in representation (1.3) and in the function $S(x)$ in representation (1.5). Note that they coincide since

$$
\begin{gathered}
\frac{\left(x-x_{j+1 / 2}\right)^{2}}{2 h^{2}}\left(f\left(x_{j}\right)-f^{\prime}\left(x_{j}\right) h\right)+\left(\frac{x_{j+1}-x}{h}-\frac{\left(x-x_{j+1 / 2}\right)^{2}}{h^{2}}\right) f\left(x_{j}\right) \\
+\left(\frac{\left(x-x_{j+1 / 2}\right)^{2}}{2 h^{2}}+\frac{x-x_{j}}{h}\right)\left(f\left(x_{j}\right)+f^{\prime}\left(x_{j}\right) h\right)=f\left(x_{j}\right)+f^{\prime}\left(x_{j}\right) .
\end{gathered}
$$

Taking this into account, we have for any $x \in\left[x_{j} ; x_{j+1 / 2}\right]$

$$
\begin{aligned}
& S(x)-f(x)=\frac{\left(x-x_{j+1 / 2}\right)^{2}}{2 h^{2}} \frac{1}{h_{1}}\left[\int_{x_{j-1}-\frac{h_{1}}{2}}^{x_{j-1}+\frac{h_{1}}{2}} \frac{1}{2} f^{\prime \prime}(t)\left(t-x_{j-1}+\frac{h_{1}}{2}\right)^{2} d t+\int_{x_{j-1}+\frac{h_{1}}{2}}^{x_{j}} f^{\prime \prime}(t) h_{1}\left(t-x_{j-1}\right) d t\right] \\
& +\left(\frac{x_{j+1}-x}{h}-\frac{\left(x-x_{j+1 / 2}\right)^{2}}{h^{2}}\right) \frac{1}{h_{1}}\left[\int_{x_{j}-\frac{h_{1}}{2}}^{x_{j}} \frac{1}{2} f^{\prime \prime}(t)\left(t-x_{j}+\frac{h_{1}}{2}\right)^{2} d t+\int_{x_{j}}^{x_{j}+\frac{h_{1}}{2}} \frac{1}{2} f^{\prime \prime}(t)\left(t-x_{j}-\frac{h_{1}}{2}\right)^{2} d t\right] \\
& +\left(\frac{\left(x-x_{j+1 / 2}\right)^{2}}{2 h^{2}}+\frac{x-x_{j}}{h}\right) \frac{1}{h_{1}}\left[\int_{x_{j}}^{x_{j+1}-\frac{h_{1}}{2}} f^{\prime \prime}(t) h_{1}\left(x_{j+1}-t\right) d t+\int_{x_{j+1}-\frac{h_{1}}{2}}^{\frac{1}{2}} f^{\prime \prime}(t)\left(t-x_{j+1}-\frac{h_{1}}{2}\right)^{2} d t\right] \\
& -\int_{x_{j}}^{x} f^{\prime \prime}(t)(x-t) d t .
\end{aligned}
$$

So, for any $x \in\left[x_{j} ; x_{j+1 / 2}\right]$, the following equality holds:

$$
\begin{gathered}
S(x)-f(x)=\int_{x_{j-1}-\frac{h_{1}}{2}}^{x_{j-1}+\frac{h_{1}}{2}} f^{\prime \prime}(t) K_{1}(x, t) d t+\int_{x_{j-1}+\frac{h_{1}}{2}}^{x_{j}} f^{\prime \prime}(t) K_{2}(x, t) d t \\
+\int_{x_{j}-\frac{h_{1}}{2}}^{x_{j}} f^{\prime \prime}(t) K_{3}(x, t) d t+\int_{x_{j}}^{x_{j+1}-\frac{h_{1}}{2}} f^{\prime \prime}(t) K_{4}(x, t) d t+\int_{x_{j}}^{x_{j}} f^{\prime \prime}(t) K_{5}(x, t) d t \\
+\int_{x_{j+1}-\frac{h_{1}}{2}}^{x_{j+1}+\frac{h_{1}}{2}} f^{\prime \prime}(t) K_{6}(x, t) d t-\int_{x_{j}}^{x} f^{\prime \prime}(t) K_{7}(x, t) d t,
\end{gathered}
$$


where

$$
\begin{gathered}
K_{1}(x, t)=\frac{\left(x-x_{j+1 / 2}\right)^{2}}{4 h^{2} h_{1}}\left(t-x_{j-1}+\frac{h_{1}}{2}\right)^{2}, \\
K_{2}(x, t)=\frac{\left(x-x_{j+1 / 2}\right)^{2}}{2 h^{2}}\left(t-x_{j-1}\right), \\
K_{3}(x, t)=\left(\frac{x_{j+1}-x}{h}-\frac{\left(x-x_{j+1 / 2}\right)^{2}}{h^{2}}\right) \frac{1}{2 h_{1}}\left(t-x_{j}+\frac{h_{1}}{2}\right)^{2}, \\
K_{4}(x, t)=\left(\frac{x_{j+1}-x}{h}-\frac{\left(x-x_{j+1 / 2}\right)^{2}}{h^{2}}\right) \frac{1}{2 h_{1}}\left(t-x_{j}-\frac{h_{1}}{2}\right)^{2}, \\
K_{5}(x, t)=\left(\frac{\left(x-x_{j+1 / 2}\right)^{2}}{2 h^{2}}+\frac{x-x_{j}}{h}\right)\left(x_{j+1}-t\right), \\
K_{6}(x, t)=\left(\frac{\left(x-x_{j+1 / 2}\right)^{2}}{2 h^{2}}+\frac{x-x_{j}}{h}\right) \frac{1}{2 h_{1}}\left(t-x_{j+1}-\frac{h_{1}}{2}\right)^{2}, \\
K_{7}(x, t)=t-x .
\end{gathered}
$$

Further proof for $x \in\left[x_{j} ; x_{j+1 / 2}\right]$ is divided into two cases: 1) $0<h_{1} \leq h$ and 2) $h<h_{1} \leq 2 h$.

Case 1). Let $0<h_{1} \leq h$. Under this, the difference $S(x)-f(x)$ for $x \in\left[x_{j}, x_{j}+\frac{h_{1}}{2}\right]$ is transformed to the form

$$
\begin{gathered}
S(x)-f(x)=\int_{x_{j-1}-\frac{h_{1}}{2}}^{x_{j-1}+\frac{h_{1}}{2}} f^{\prime \prime}(t) K_{1}(x, t) d t+\int_{x_{j-1}+\frac{h_{1}}{2}}^{x_{j}-\frac{h_{1}}{2}} f^{\prime \prime}(t) K_{2}(x, t) d t \\
+\int_{x_{j}-\frac{h_{1}}{2}}^{x_{j}} f^{\prime \prime}(t)\left(K_{2}(x, t)+K_{3}(x, t)\right) d t+\int_{x_{j}}^{x} f^{\prime \prime}(t)\left(K_{4}(x, t)+K_{5}(x, t)+K_{7}(x, t)\right) d t \\
+\int_{x}^{x_{j}+\frac{h_{1}}{2}} f^{\prime \prime}(t)\left(K_{4}(x, t)+K_{5}(x, t)\right) d t+\int_{x_{j}+\frac{h_{1}}{2}}^{x_{j+1}-\frac{h_{1}}{2}} f^{\prime \prime}(t) K_{5}(x, t) d t+\int_{x_{j+1}-\frac{h_{1}}{2}}^{x_{j+1}+\frac{h_{1}}{2}} f^{\prime \prime}(t) K_{6}(x, t) d t .
\end{gathered}
$$

But for $x \in\left[x_{j}+\frac{h_{1}}{2}, x_{j+1 / 2}\right]$, its form is

$$
\begin{gathered}
S(x)-f(x)=\int_{x_{j-1}-\frac{h_{1}}{2}}^{x_{j-1}+\frac{h_{1}}{2}} f^{\prime \prime}(t) K_{1}(x, t) d t+\int_{x_{j-1}+\frac{h_{1}}{2}}^{x_{j}-\frac{h_{1}}{2}} f^{\prime \prime}(t) K_{2}(x, t) d t \\
+\int_{x_{j}-\frac{h_{1}}{2}}^{x_{j}} f^{\prime \prime}(t)\left(K_{2}(x, t)+K_{3}(x, t)\right) d t+\int_{x_{j}}^{x_{j}+\frac{h_{1}}{2}} f^{\prime \prime}(t)\left(K_{4}(x, t)+K_{5}(x, t)+K_{7}(x, t)\right) d t \\
+\int_{x_{j}+\frac{h_{1}}{2}}^{x} f^{\prime \prime}(t)\left(K_{5}(x, t)+K_{7}(x, t)\right) d t+\int_{x}^{x_{j+1}-\frac{h_{1}}{2}} f^{\prime \prime}(t) K_{5}(x, t) d t+\int_{x_{j+1}-\frac{h_{1}}{2}}^{x_{j+1}+\frac{h_{1}}{2}} f^{\prime \prime}(t) K_{6}(x, t) d t .
\end{gathered}
$$


To obtain the estimation value on the function class $W_{\infty}^{2}=W_{\infty}^{2}(\mathbb{R})$, we shall prove that

under $x \in\left[x_{j}, x_{j+1 / 2}\right]: \quad K_{1}(x, t) \geq 0$ for $t \in\left[x_{j-1}-\frac{h_{1}}{2} ; x_{j-1}+\frac{h_{1}}{2}\right], \quad K_{2}(x, t) \geq 0$ for $t \in\left[x_{j-1}+\frac{h_{1}}{2} ; x_{j}-\frac{h_{1}}{2}\right], \quad K_{2}(x, t)+K_{3}(x, t) \geq 0$ for $t \in\left[x_{j}-\frac{h_{1}}{2} ; x_{j}\right], \quad K_{6}(x, t) \geq 0$ for $t \in\left[x_{j+1}-\frac{h_{1}}{2} ; x_{j+1}+\frac{h_{1}}{2}\right]$

under $x \in\left[x_{j}, x_{j}+\frac{h_{1}}{2}\right]: K_{4}(x, t)+K_{5}(x, t)+K_{7}(x, t) \geq 0$ for $t \in\left[x_{j} ; x\right], K_{4}(x, t)+K_{5}(x, t) \geq 0$ for $t \in\left[x ; x_{j}+\frac{h_{1}}{2}\right], K_{5}(x, t) \geq 0$ for $t \in\left[x_{j}+\frac{h_{1}}{2} ; x_{j+1}-\frac{h_{1}}{2}\right]$;

under $x \in\left[x_{j}+\frac{h_{1}}{2}, x_{j+1 / 2}\right]: K_{4}(x, t)+K_{5}(x, t)+K_{7}(x, t) \geq 0$ for $t \in\left[x_{j} ; x_{j}+\frac{h_{1}}{2}\right], K_{5}(x, t)+$ $K_{7}(x, t) \geq 0$ for $t \in\left[x_{j}+\frac{h_{1}}{2} ; x\right], K_{5}(x, t) \geq 0$ for $t \in\left[x ; x_{j+1}-\frac{h_{1}}{2}\right]$.

All these inequalities (except only two) immediately follow from definitions of the functions $K_{j}(x, t) \quad(j=\overline{1,7})$. So, it is only necessary to verify that $K_{4}(x, t)+K_{5}(x, t)+K_{7}(x, t) \geq 0$ for $x \in\left[x_{j}, x_{j}+\frac{h_{1}}{2}\right], t \in\left[x_{j} ; x\right]$, for $x \in\left[x_{j}+\frac{h_{1}}{2}, x_{j+1 / 2}\right], t \in\left[x_{j} ; x_{j}+\frac{h_{1}}{2}\right]$ and $K_{5}(x, t)+K_{7}(x, t) \geq 0$ for $x \in\left[x_{j}+\frac{h_{1}}{2}, x_{j+1 / 2}\right], t \in\left[x_{j}+\frac{h_{1}}{2} ; x\right]$.

The function $K_{4}(x, t)+K_{5}(x, t)+K_{7}(x, t)$ is the quadratic trinomial in the variable $t$ with the positive leading coefficient; at the ends of the intervals $\left[x_{j} ; x\right],\left[x_{j} ; x_{j}+\frac{h_{1}}{2}\right]$ this function (as one of the variable $t$ ) takes the positive values, and abscissa of the corresponding parabola apex is placed at the left from the point $x_{j}$. From this, the non-negativity of this function on the mentioned sets follows.

The function $K_{5}(x, t)+K_{7}(x, t)$ is linear in the variable $t$ and takes non-negative values at the ends of the interval $\left[x_{j}+\frac{h_{1}}{2} ; x\right]$. Hence, $K_{5}(x, t)+K_{7}(x, t) \geq 0$ in the whole interval $\left[x_{j}+\frac{h_{1}}{2} ; x\right]$ for $x \in\left[x_{j}+\frac{h_{1}}{2}, x_{j+1 / 2}\right]$.

Taking into account the above proved facts, it follows from formulas (2.3) and (2.4) that to obtain the estimate from above for the value $|S(x)-f(x)|$ (for these formulas) in the class $W_{\infty}^{2}(\mathbb{R})$ and, hence, in formula (2.1), the function $f^{\prime \prime}(t)$ can be substituted by 1 in (2.3) and (2.4).

Put $f^{\prime \prime}(t)=1$ and calculate for it values of integrals in the right-hand side of formula $(2.1)$; denote this value by the symbol $J$. After elementary calculations, we obtain that $J=\frac{h^{2}}{8}+\frac{h_{1}^{2}}{24}$. It implies that the exact inequality

$$
|f(x)-S(x)| \leq \frac{h^{2}}{8}+\frac{h_{1}^{2}}{24}
$$

holds for any function $f \in W_{\infty}^{2}\left[x_{j-1}-\frac{h_{1}}{2} ; x_{j+1}+\frac{h_{1}}{2}\right]$ for any $x \in\left[x_{j} ; x_{j+1 / 2}\right]$. Moreover, the sign of equality is provided by the function $f(t)=\frac{t^{2}}{2}$ for $t \in\left[x_{j-1}-\frac{h_{1}}{2} ; x_{j+1}+\frac{h_{1}}{2}\right]$.

Similarly, for the function $f \in W_{\infty}^{2}\left[x_{j}-\frac{h_{1}}{2} ; x_{j+2}+\frac{h_{1}}{2}\right]$ for any $x \in\left[x_{j+1 / 2} ; x_{j+1}\right]$, we derive the exact inequality

$$
|f(x)-S(x)| \leq \frac{h^{2}}{8}+\frac{h_{1}^{2}}{24},
$$

and the sign of equality is provided by the function $f(t)=\frac{t^{2}}{2}$ for $t \in\left[x_{j}-\frac{h_{1}}{2} ; x_{j+2}+\frac{h_{1}}{2}\right]$. 
Case 2). Let $h<h_{1} \leq 2 h$. In this case, the difference $S(x)-f(x)$ for $x \in\left[x_{j}, x_{j+1}-\frac{h_{1}}{2}\right]$ is transformed to the form

$$
\begin{aligned}
& S(x)-f(x)=\int_{x_{j-1}-\frac{h_{1}}{2}}^{x_{j}-\frac{h_{1}}{2}} f^{\prime \prime}(t) K_{1}(x, t) d t+\int_{x_{j}-\frac{h_{1}}{2}}^{x_{j-1}+\frac{h_{1}}{2}} f^{\prime \prime}(t)\left(K_{1}(x, t)+K_{3}(x, t)\right) d t \\
& +\int_{x_{j-1}+\frac{h_{1}}{2}}^{x_{j}} f^{\prime \prime}(t)\left(K_{2}(x, t)+K_{3}(x, t)\right) d t+\int_{x_{j}}^{x} f^{\prime \prime}(t)\left(K_{4}(x, t)+K_{5}(x, t)+K_{7}(x, t)\right) d t \\
& +\int_{x}^{x_{j+1}-\frac{h_{1}}{2}} f^{\prime \prime}(t)\left(K_{4}(x, t)+K_{5}(x, t)\right) d t+\int_{x_{j+1}-\frac{h_{1}}{2}}^{x_{j}+\frac{h_{1}}{2}} f^{\prime \prime}(t)\left(K_{4}(x, t)+K_{6}(x, t)\right) d t \\
& +\int_{x_{j}+\frac{h_{1}}{2}}^{x_{j+1}+\frac{h_{1}}{2}} f^{\prime \prime}(t) K_{6}(x, t) d t \\
& S(x)-f(x)=\int_{x_{j-1}-\frac{h_{1}}{2}}^{x_{j}-\frac{h_{1}}{2}} f^{\prime \prime}(t) K_{1}(x, t) d t+\int_{x_{j}-\frac{h_{1}}{2}}^{x_{j-1}+\frac{h_{1}}{2}} f^{\prime \prime}(t)\left(K_{1}(x, t)+K_{3}(x, t)\right) d t \\
& +\int_{x_{j-1}+\frac{h_{1}}{2}}^{x_{j}} f^{\prime \prime}(t)\left(K_{2}(x, t)+K_{3}(x, t)\right) d t+\int_{x_{j}}^{x_{j+1}-\frac{h_{1}}{2}} f^{\prime \prime}(t)\left(K_{4}(x, t)+K_{5}(x, t)+K_{7}(x, t)\right) d t \\
& +\int_{x_{j+1}-\frac{h_{1}}{2}}^{x} f^{\prime \prime}(t)\left(K_{4}(x, t)+K_{6}(x, t)+K_{7}(x, t)\right) d t+\int_{x}^{x_{j}+\frac{h_{1}}{2}} f^{\prime \prime}(t)\left(K_{4}(x, t)+K_{6}(x, t)\right) d t \\
& +\int_{x_{j}+\frac{h_{1}}{2}}^{x_{j+1}+\frac{h_{1}}{2}} f^{\prime \prime}(t) K_{6}(x, t) d t
\end{aligned}
$$

To obtain the error estimate in the class $W_{\infty}^{2}(\mathbb{R})$, prove that

under $x \in\left[x_{j}, x_{j+1 / 2}\right]$, the following inequalities hold: $K_{1}(x, t) \geq 0$ for $t \in\left[x_{j-1}-\frac{h_{1}}{2} ; x_{j}-\frac{h_{1}}{2}\right]$, $K_{1}(x, t)+K_{3}(x, t) \geq 0$ for $t \in\left[x_{j}-\frac{h_{1}}{2} ; x_{j-1}+\frac{h_{1}}{2}\right], K_{2}(x, t)+K_{3}(x, t) \geq 0$ for $t \in\left[x_{j-1}+\frac{h_{1}}{2} ; x_{j}\right]$, $K_{6}(x, t) \geq 0$ for $t \in\left[x_{j}+\frac{h_{1}}{2} ; x_{j+1}+\frac{h_{1}}{2}\right]$;

under $x \in\left[x_{j}, x_{j+1}-\frac{h_{1}}{2}\right]$ these inequalities are: $K_{4}(x, t)+K_{5}(x, t)+K_{7}(x, t) \geq 0$ for $t \in\left[x_{j} ; x\right]$, $K_{4}(x, t)+K_{5}(x, t) \geq 0$ for $t \in\left[x ; x_{j+1}-\frac{h_{1}}{2}\right], K_{4}(x, t)+K_{6}(x, t) \geq 0$ for $t \in\left[x_{j+1}-\frac{h_{1}}{2} ; x_{j}+\frac{h_{1}}{2}\right]$; 
under $x \in\left[x_{j+1}-\frac{h_{1}}{2}, x_{j+1 / 2}\right]$ the inequalities hold: $K_{4}(x, t)+K_{5}(x, t)+K_{7}(x, t) \geq 0$ for $t \in$ $\left[x_{j} ; x_{j+1}-\frac{h_{1}}{2}\right], K_{4}(x, t)+K_{6}(x, t)=K_{7}(x, t) \geq 0$ for $t \in\left[x_{j+1}-\frac{h_{1}}{2} ; x\right]$, and $K_{4}(x, t)+K_{6}(x, t) \geq 0$ for $t \in\left[x ; x_{j}+\frac{h_{1}}{2}\right]$.

All these inequalities (except two ones) immediately follow from definitions of the function $K_{j}(x, t) \quad(j=\overline{1,7})$. It is only necessary to verify that $K_{4}(x, t)+K_{5}(x, t)+K_{7}(x, t) \geq 0$ under $x \in\left[x_{j}, x_{j+1}-\frac{h_{1}}{2}\right], t \in\left[x_{j} ; x\right]$ and under $x \in\left[x_{j+1}-\frac{h_{1}}{2}, x_{j+1 / 2}\right], t \in\left[x_{j} ; x_{j+1}-\frac{h_{1}}{2}\right]$, and $K_{4}(x, t)+K_{6}(x, t)+K_{7}(x, t) \geq 0$ under $x \in\left[x_{j+1}-\frac{h_{1}}{2}, x_{j+1 / 2}\right], t \in\left[x_{j+1}-\frac{h_{1}}{2} ; x\right]$.

The function $K_{4}(x, t)+K_{5}(x, t)+K_{7}(x, t)$ is the quadratic trinomial in the variable $t$ with the positive leading coefficient; at the ends of the intervals $\left[x_{j} ; x\right],\left[x_{j} ; x_{j+1}-\frac{h_{1}}{2}\right]$, this trinomial takes positive values, and abscissa of the corresponding parabola apex is placed at the left from the point $x_{j}$.

The function $K_{4}(x, t)+K_{6}(x, t)+K_{7}(x, t)$ for $x \in\left[x_{j+1}-\frac{h_{1}}{2} ; x_{j+1 / 2}\right], t \in\left[x_{j+1}-\frac{h_{1}}{2} ; x\right]$ possesses the same properties. Remind that this function is also the quadratic trinomial in the variable $t$ with the positive leading coefficient; at the ends of the interval $\left[x_{j+1}-\frac{h_{1}}{2} ; x\right]$, this trinomial takes positive values, and abscissa of the corresponding parabola apex is placed at the left from the point $x_{j+1}-\frac{h_{1}}{2}$. It implies non-negativity of the considered functions in the mentioned sets.

Taking into account the above proved facts, it follows from formulas (2.5) and (2.6) that to obtain the estimate from above for the value $|S(x)-f(x)|$ (for these formulas) in the class $W_{\infty}^{2}(\mathbb{R})$ and, hence, in formula (2.1), the function $f^{\prime \prime}(t)$ can be substituted by 1 .

Put $f^{\prime \prime}(t)=1$ and calculate for it values of integrals in the right-hand side of formula (2.1); denote this value by the symbol $J$. After elementary calculations, we obtain that $J=\frac{h^{2}}{8}+\frac{h_{1}^{2}}{24}$. It implies that the exact inequality

$$
|f(x)-S(x)| \leq \frac{h^{2}}{8}+\frac{h_{1}^{2}}{24}
$$

holds for any function $f \in W_{\infty}^{2}\left[x_{j-1}-\frac{h_{1}}{2} ; x_{j+1}+\frac{h_{1}}{2}\right]$ under any $x \in\left[x_{j} ; x_{j+1 / 2}\right]$. Moreover, the equality sign is provided by the function $f(t)=\frac{t^{2}}{2}$ for $t \in\left[x_{j-1}-\frac{h_{1}}{2} ; x_{j+1}+\frac{h_{1}}{2}\right]$.

Similarly, for the function $f \in W_{\infty}^{2}\left[x_{j}-\frac{h_{1}}{2} ; x_{j+2}+\frac{h_{1}}{2}\right]$ under any $x \in\left[x_{j+1 / 2} ; x_{j+1}\right]$, we derive the exact inequality

$$
|S(x)-f(x)| \leq \frac{h^{2}}{8}+\frac{h_{1}^{2}}{24}
$$

and the equality sign is provided by the function $f(t)=\frac{t^{2}}{2}$ for $t \in\left[x_{j}-\frac{h_{1}}{2} ; x_{j+2}+\frac{h_{1}}{2}\right]$.

\section{Acknowledgements}

The Author expresses deep gratitudes to Prof. K. Etter (Germany) and to Dr. V.L. Miroshnichenko (Novosibirsk, Russia) for the fruitful discussions of the results obtained. 


\section{REFERENCES}

1. Zavyalov Yu.S., Kvasov B. I., Miroshnichenko V. L. Spline-functions methods. Moscow: Nauka, 1980. 355 p. [in Russian]

2. Piegl L., Tiller W. The NURBS Book. New York: Springer, 1997. 646 p.

3. Zavyalov Yu.S. On formulas of local approximation exact on the cubic splines // Comp. systems, 1998. Vol. 128. P. 75-88. [in Russian]

4. Korneychuk N.P. Splines in the approximation theory. Moscow: Nauka, 1984. 352 p. [in Russian]

5. Subbotin Yu.N. Heritance of monotonisity and convexity properties under local approximation // J. Comp. Math. and Math. Physics, 1993. Vol. 37, no. 7. P. 996-1003. [in Russian]

6. Subbotin Yu.N. Extremal problems of functional interpolation and interpolation of splines in the mean // Trudy Steklov Math. Institute of RAS, 1975. Vol. 109. P. 35-60. [in Russian]

7. Subbotin Yu.N. Extremal functional interpolation in the mean with the minimal value of the n-th derivative on large intervals of meaning // Math. zametki, 1996. Vol. 59, no. 1. P. 114-132. [in Russian]

8. Subbotin Yu.N. Extremal $L_{p}$-interpolation in the mean on intersecting intervals of meaning // Izv. RAS Ser. Math., 1997. Vol. 61, no. 1. P. 177-198. [in Russian] DOI: https://doi.org/104213/im110

9. Shevaldin V.T. Some problems of extremal interpolation in the mean for linear differential operators // Trudy Steklov Math. Institute of RAS, 1983. Vol. 164. P. 203-240. [in Russian]

10. Shevaldin V.T. Extremal interpolation in the mean on intersecting intervals of meaning and $L$-splines // Izv. RAS Ser. Math., 1998. Vol. 62, no. 4. P. 201-224. [in Russian] DOI: https://doi.org/104213/im193 